\title{
INTERPOLATION OF THE DILUTION GRID IN THE BENGAL CODE
}

\author{
Nathan A. Gibson and Steven J. Douglass \\ Naval Nuclear Laboratory \\ P.O. Box 1072, Schenectady, NY 12301-1072, USA \\ nathan.gibson@unnpp.gov, steven.douglass@unnpp.gov
}

\begin{abstract}
In the implementation of the equivalence-in-dilution self-shielding method, multigroup cross sections as a function of the background cross section (i.e., the dilution) are needed. The background cross section of a particular nuclide in a particular material is determined iteratively based on geometry and material composition, resulting in a large number of cross section look-ups and a continuously varying dilution as the independent variable. Typically, multigroup cross sections are interpolated based on a reference grid of a set of dilution values and corresponding cross sections. The selection of this grid and the interpolant used between the grid are not well-documented in the literature, and so the approach used by the Bengal code is of note to the technical community. This work compares the interpolation scheme of the legacy code TRANSX to a newly developed interpolation scheme based on cubic Hermite splines, both by looking at the relative error in generated cross sections and by assessing the impact on a simple reactor simulation.
\end{abstract}

KEYWORDS: interpolation; self-shielding; multigroup cross sections

\section{INTRODUCTION}

Bengal [1] is a multigroup cross section processing code used primarily with the Jaguar [2] discrete ordinates transport code and the Lynx code executor for production-level analysis at the Naval Nuclear Laboratory. Bengal uses the equivalence-in-dilution (or Bondarenko) self-shielding method, which relies on multigroup constants as a function of the background cross section (i.e., the dilution). Bengal takes the typical approach of tabulating multigroup constants at a select set of reference values of the background cross section and performing interpolation for intermediate values. The selection of these reference points and the methods used for interpolation have not been well-documented in the literature, and therefore the approach chosen and the supporting analysis for Bengal is worthy of note for the technical community.

Bengal was developed as a replacement of the TRANSX code [3], originally seeking to mimic its capabilities in a more modern and extensible code base, with a focus on performance for large-scale calculations. Thus, Bengal initially used the same interpolation scheme as TRANSX. However, as this paper will show, this scheme was found to have several drawbacks, and a new approach to interpolation was adopted. Because Bengal requires both accuracy and efficiency to operate within a production environment, the considerations used to assess options to represent the dependence of the cross sections on dilution included the following: an acceptable level of interpolation error; 
a relatively small number of reference points; computational efficiency; a common grid for all nuclides, energy groups, and reaction channels; and no manipulation needed by downstream users.

\section{THEORY AND BACKGROUND}

Bengal performs resonance self-shielding calculations via the equivalence-in-dilution method, also known as the Bondarenko method. Presented here is a very brief summary of the theory. A much more complete description can be found in [4] or almost any reactor physics textbook, such as [5].

In a system of neutrons slowing down in an infinite homogeneous medium comprised of a resonant nuclide and a moderator, the reaction rate (and therefore the multigroup cross section) due to the presence of a single resonance is a function of the amount of admixed moderating material. When there is relatively little moderation compared to the amount of resonant material, resonances exhibit a self-shielding effect; that is, resonances cause a depression in the neutron flux at the resonance energy, lowering the reaction rate. The parameter used to measure the relative amount of moderation compared to the resonant material is typically called the background cross section $\sigma_{b}$ or the dilution. In more realistic geometries, the effect of a lumped resonant region surrounded by a moderated region can be captured treating the resonant region as an equivalent infinite medium with an increased background cross section. The simplest approach to defining the dilution of this equivalent system uses Wigner's Rational Approximation, and the background cross section is

$$
\sigma_{b}=\frac{N^{+} \sigma^{+}}{N^{*}}+\frac{1-C}{N^{*} \bar{l}}
$$

where $N$ is the number density, $\sigma$ is the cross section, + and $*$ represent the moderator and resonant material, respectively, $C$ is the Dancoff factor (representing the effect of repeating geometry), and $\bar{l}$ is the mean-chord length.

In real applications, there are likely more than one resonant nuclide in a material region, both at beginning of life (e.g., multiple isotopes of uranium) and after depletion (e.g., transmuted actinides). Furthermore, even non-resonant nuclides do not have the constant cross section assumed in the simple theory. Bengal uses a common iterative approach, replacing Eq. (1) with

$$
\sigma_{b}^{i}=\frac{1}{N^{i}} \sum_{j \neq i} N^{j} \sigma^{j}+\frac{1-C}{N^{i} \bar{l}},
$$

where the superscripts indicate the index of the nuclide in the material region. Note that the background cross section is different for each nuclide in the material and that this approach requires iteration, as the cross section of each nuclide depends on that of each other nuclide.

Cross sections in Bengal libraries are generated as a function of the background cross section using the GROUPR module of NJOY [6] and are first pre-processed by the MATXSR module. Continuousenergy cross sections are collapsed to multigroup using an assumed flux. The simplest flux to use is that of the narrow resonance approximation, which assumes

$$
\phi(E)=\frac{1}{E} \frac{\sigma_{p}+\sigma_{b}}{\sigma_{t}(E)+\sigma_{b}},
$$

where $\phi(E)$ is the flux spectrum, $\sigma_{p}$ and $\sigma_{t}$ are the potential scattering and total cross sections of the nuclide of interest, and $\sigma_{b}$ is the background cross section. Alternatively, NJOY provides an 
option to use the built-in flux calculator, which is better suited to treat large low-lying resonances in nuclides such as U-238.

If enough background cross section values are used, the result of this process is a multigroup cross section as a smooth function of the background cross section. When plotted with a logarithmic abscissa, this function looks very similar to a hyperbolic tangent function. Note that, depending upon the resonance structure of the underlying continuous energy cross section, these tanh-like functions have differing maxima and minima, locations of inflection points, and slopes near the inflection points.

Because these curves must be kept for each nuclide, for each group, and for each reaction type, it is desired to use a small number of points to represent the curve and to interpolate for values intermediate to the points. The points chosen are collectively referred to as the dilution grid. The TRANSX interpolation scheme that was originally adopted by Bengal uses linear interpolation based on $\log \left(\sigma_{b}\right)$ (log-linear) for small values of the background cross section and linear interpolation based on $1 / \sigma_{b}$ (inverse-linear) for large values. The TRANSX manual notes that this interpolation scheme was selected for its reliability and stability, but requires more points in the dilution grid than the scheme in older TRANSX versions, which used a combination of $1 / \sigma_{b}$, Langrangian, and quadratic interpolation.

For all analysis shown in this paper, the XMAS 172-group energy group structure [7] is used.

\section{INTERPOLATION ERRORS}

Consider first a naive approach to interpolation, using the interpolation scheme of TRANSX (loglinear for background cross sections below $10^{4} \mathrm{~b}$ and inverse-linear for larger values) with the seven-point dilution grid of Table 1. While this scheme results in relatively small errors for most nuclides, reaction types, and energy groups, its shortcomings are easily seen with cross sections featuring large resonances. Figure 1 graphically shows the difference between interpolated values and a reference solution for the 33.72-37.27 eV energy group for the U-238 total cross section. Large errors are easily seen between $10^{2} \mathrm{~b}$ and $10^{3} \mathrm{~b}$, where the log-linear interpolation does not capture the curvature of the function, and above $10^{4} \mathrm{~b}$, where the inverse-linear interpolation is used prior to the cross section reaching its asymptotic value. The first plot of Fig. 2 shows the relative error in interpolation for all the resonant groups for U-238, where the largest errors are between $20 \%$ and $30 \%$ and several groups exhibit errors on the order of $10 \%$. Also note that due to the convexity of the curve at low values of the dilution cross section, the log-linear interpolation systematically overpredicts compared to the reference. In order to reduce these errors, there are two logical approaches: increase the number of base points in the interpolation, and use an interpolant that better captures the shape of the underlying function.

When increasing the number of base points, this analysis quickly settled upon 15 points being the best option. There is a desire to minimize the number of base points, as the size of the multigroup library in both memory usage and disk storage is typically directly proportional to the number of points. Furthermore, there is an apparent limit of 15 dilution values that can be processed by the MATXSR module of NJOY. Although more values could be obtained through multiple executions of NJOY, this would be a challenge to implement in a production system and was not deemed 
Table 1: Dilution grids used in this analysis. On the left, a naively chosen seven-point dilution grid. On the right, a fifteen-point dilution grid based on approximately equally logarithmically spaced values.

\begin{tabular}{ccc}
\hline \multicolumn{3}{c}{ Background Cross Section $[\mathrm{b}]$} \\
\hline Seven-Point Grid & \multicolumn{3}{c}{ Fifteen-Point Grid } \\
\hline $1.0 \mathrm{E}+10$ & $1.0 \mathrm{E}+10$ & $3.0 \mathrm{E}+02$ \\
$1.0 \mathrm{E}+04$ & $1.0 \mathrm{E}+06$ & $1.0 \mathrm{E}+02$ \\
$1.0 \mathrm{E}+03$ & $3.0 \mathrm{E}+05$ & $3.0 \mathrm{E}+01$ \\
$1.0 \mathrm{E}+02$ & $1.0 \mathrm{E}+05$ & $1.0 \mathrm{E}+01$ \\
$1.0 \mathrm{E}+01$ & $3.0 \mathrm{E}+04$ & $3.0 \mathrm{E}+00$ \\
$1.0 \mathrm{E}+00$ & $1.0 \mathrm{E}+04$ & $1.0 \mathrm{E}+00$ \\
$1.0 \mathrm{E}-01$ & $3.0 \mathrm{E}+03$ & $1.0 \mathrm{E}-01$ \\
& $1.0 \mathrm{E}+03$ & \\
\hline
\end{tabular}

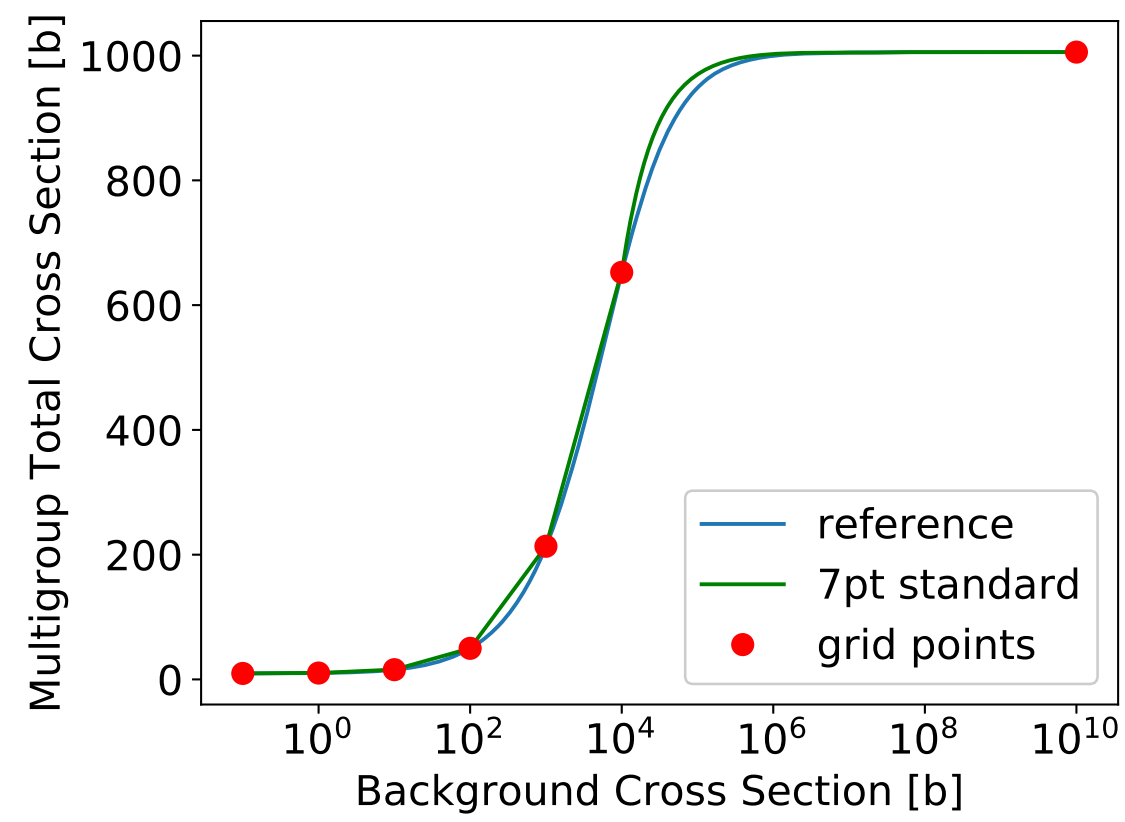

Figure 1: Comparison of a naive interpolation scheme to a reference curve for the U-238 cross section in an energy group containing the $36.6 \mathrm{eV}$ resonance. Deviations are clearly seen at the elbows. 

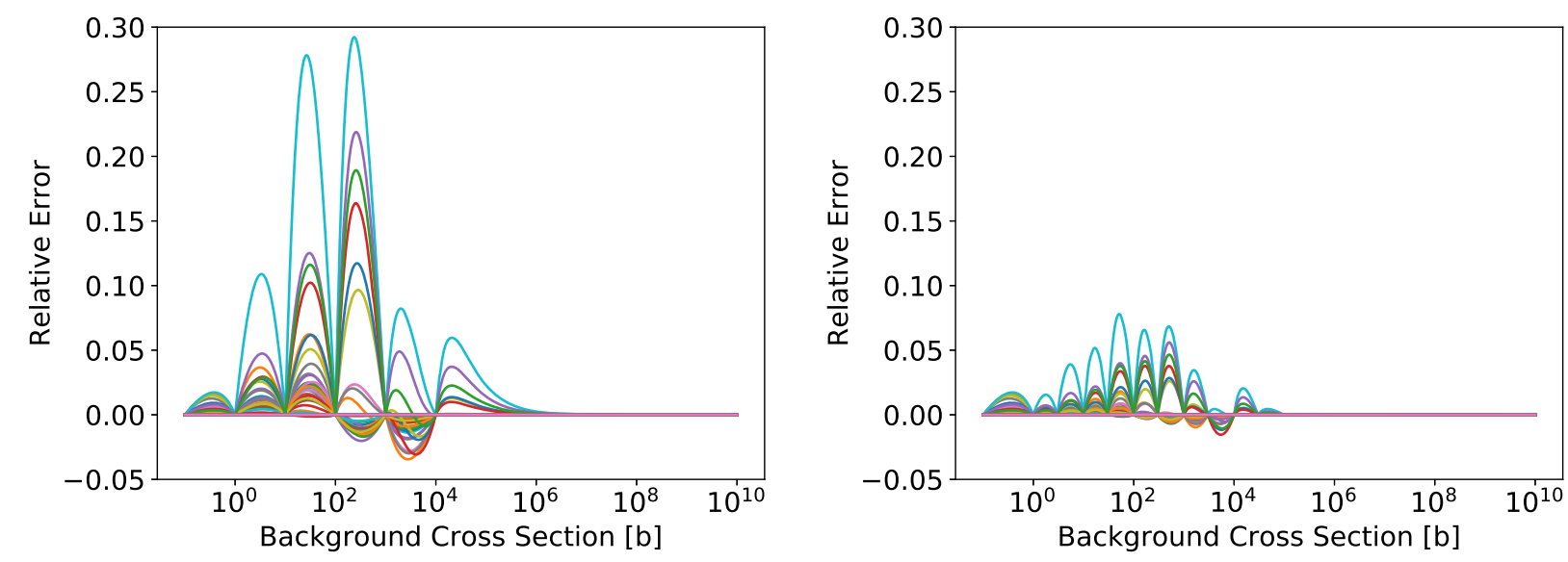

Figure 2: Relative error of interpolated cross sections for each resonant energy group of U-238 in the XMAS 172-group structure using the 7-point dilution grid (left) and the 15-point dilution grid (right) and the TRANSX interpolation scheme.

necessary. Because adequate interpolation error was attainable with 15 points, using additional base points were not pursued.

One possible 15-point dilution grid is shown in Table 1. This grid was selected simply by using approximately logarithmically equally spaced background cross section values across the range of values for which cross sections are typically sensitive. Note that, as shown in Section 4, this grid is not ideal for typical light water reactor problems, as there are not base-points near U-238's typical dilution values. However, maximum cross section errors due to interpolation are not found to be a strong function of the placement of the base points, and this simple choice highlights another consideration that those implementing equivalence-in-dilution must face. A plot of the error across all energy groups using the interpolation scheme from TRANSX and the 15-point dilution grid is given in the second plot of Fig. 2. Although errors are reduced, the remaining 5\%-10\% errors are still unreasonably high for interpolation.

In order to obtain acceptable levels of interpolation error without refining the dilution grid further, other interpolants were investigated. Some of the options considered and ultimately not selected are discussed briefly in Section 3.1. Ultimately, a combination of cubic Hermite splines and inverse-linear interpolation was selected as the approach. Cubic splines are piece-wise third-order polynomials with coefficients chosen such that the pieces have continuous first derivatives at the base points, which are often called knots. The formulation chosen for use here is with polynomials of $\log \left(\sigma_{b}\right)$ in Hermite form. The cubic Hermite spline is given by

$$
\begin{aligned}
p(x)= & h_{00}(t) p_{k}+h_{10}(t)\left(x_{k+1}-x_{k}\right) m_{k}+ \\
& h_{01}(t) p_{k+1}+h_{11}(t)\left(x_{k+1}-x_{k}\right) m_{k+1} \quad x \in\left[x_{k}, x_{k+1}\right],
\end{aligned}
$$

where $p$ is the function being interpolated (multigroup cross section), $p_{k}$ is the function's value at knot $k, x$ is the independent variable $\left(\log \left(\sigma_{b}\right)\right), x_{k}$ is the position of knot $k, t$ is a scaling function of $x, m_{k}$ is the slope of the spline at knot $k$, and $h$ functions are basis polynomials of $t$. The scaling 
function is given by

$$
t=\frac{x-x_{k}}{x_{k+1}-x_{k}}
$$

and the basis polynomials are given by

$$
\begin{aligned}
& h_{00}(t)=2 t^{3}-3 t^{2}+1 \\
& h_{10}(t)=t^{3}-2 t^{2}+t \\
& h_{01}(t)=-2 t^{3}+3 t^{2} \\
& h_{11}(t)=t^{3}-t^{2} .
\end{aligned}
$$

The derivatives $m$ are free parameters. Many spline implementations choose the derivatives at interior knots such that the second derivatives are continuous. This requires the inversion of a $K \times K$ matrix, with $K$ being the number of knots. This approach was found to be overly costly and unnecessary for this application. Instead, centered finite differences were used to approximate the derivatives at interior knots:

$$
m_{k}=\frac{1}{2} \frac{p_{k}-p_{k-1}}{x_{k}-x_{k-1}}+\frac{1}{2} \frac{p_{k+1}-p_{k}}{x_{k+1}-x_{k}}
$$

Rather than using one-sided derivatives at the ends of the spline, derivatives were taken to be 0 , using knowledge of the shape of the dilution curve.

Note that when using spline interpolation as is defined here, the property that partial cross sections sum to the total cross section is conserved. This is one advantage of spline interpolation over other advanced interpolation procedures. In general, this property will not be maintained if the scheme is nonlinear in the dependent variable.

Although splines implemented in this fashion with the 15-point dilution grid yielded acceptable interpolation errors for most nuclides and groups, larger errors with dilutions above $10^{6} \mathrm{~b}$ were observed for nuclides and groups with large resonances (e.g., U-238's low-lying resonances) due to the finite difference being a poor approximation for the derivative at $10^{6} \mathrm{~b}$. To rectify this, Bengal's spline interpolation switches to inverse-linear interpolation between the top two dilution values. A plot of the error for U-238's resonant groups using this interpolation scheme is shown in Fig. 3. Errors only exceed $1 \%$ for one group in one range of background cross sections. Although $1 \%$ and large fractions thereof are still relatively large interpolation errors, it was found that this error did not greatly impact downstream calculations and could easily be corrected through global normalization procedures (e.g., equivalence theory).

\subsection{Other Possibilities}

During the course of work leading to the interpolation scheme detailed here, several other options were considered. For instance, other interpolation schemes such as log-log and log-quadratic were investigated. These interpolation schemes did not provide lower error estimates than splines provided. Furthermore, because these schemes were not linear in the dependent variable, there was no guarantee that interpolation of the total cross section would yield the same value as the sum of interpolated partial cross sections, thus adding an additional source of error to the problem at hand. 


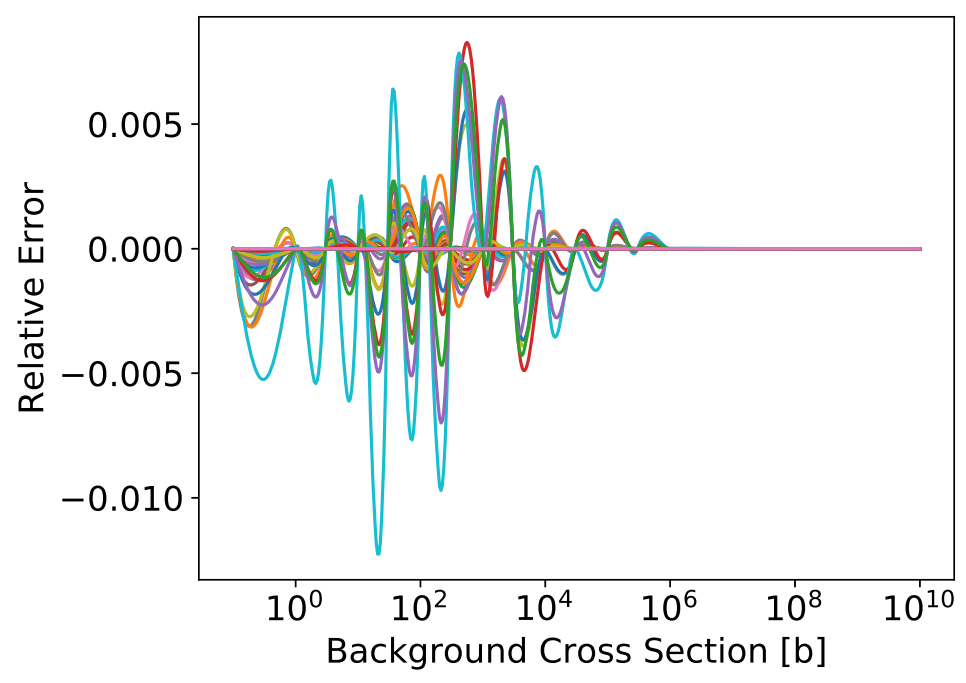

Figure 3: Relative error of interpolated cross sections for each resonant energy group of U-238 in the XMAS 172-group structure using the 15-point dilution grid and the proposed spline-based interpolation scheme.

Alternatively, functional representation of the dilution curve was considered. Here, the reference points computed by NJOY were used with an optimization process to determine the needed parameters. One functional representation considered was the hyperbolic tangent function, formulated as

$$
\sigma\left(\sigma_{b}\right)=\alpha_{1} \tanh \left(\alpha_{2} \log \sigma_{b}+\alpha_{3}\right)+\alpha_{4},
$$

both with $\alpha_{1}$ and $\alpha_{4}$ left free for the optimizer and with $\alpha_{1}$ and $\alpha_{4}$ being determined analytically. While this were found to perform adequately for most nuclides and energy groups, groups with particularly large resonances, such as the U-238 $36.6 \mathrm{eV}$ resonance considered previously, were not well-represented. When plotted, departure between the reference values and the functional fit can easily be seen near the knees of the function.

A fitting function based on the hyperbolic tangent is equivalent to a shifted and scaled sigmoid or logistic function. This was considered a good candidate as a fitting function, as when using $\log \sigma_{b}$, the sigmoid function takes the form

$$
f\left(\sigma_{b}\right)=\sigma_{b} /\left(1+\sigma_{b}\right),
$$

which is reminiscent of the narrow resonance flux given in Eq. (3). No significant improvements were found by varying the parameterization of logistic functions.

However, functional representations may indeed still be possible, as this was not an exhaustive study. Sums of hyperbolic tangents or logistic functions (which would be analogous to multiterm rational expansions for the escape probability [8], and several other functions with a similar " $S$ "-shaped behavior (e.g., the error function) could likely prove to be more robust. However, the difficulty in fitting more complicated functions combined with the interpolation approach selected by this study being deemed sufficient led the authors to not pursue functional fits any further. 
Table 2: Comparison of eigenvalues of the VERA pin-cell problem with depletion. The $50 \mathrm{~b}$ results are characteristic of the pin-cell, but have an exaggerated error due to the location of base-points in the dilution grids. The $100 \mathrm{~b}$ results show much smaller errors due to the grids containing a base point near the adjusted U-238 dilution.

\begin{tabular}{cccccccc}
\hline Burnup & \multicolumn{3}{c}{$50 \mathrm{~b}$ Dilution } & & \multicolumn{3}{c}{$100 \mathrm{~b}$ Dilution } \\
\cline { 2 - 3 } \cline { 7 - 8 }$[\mathrm{MWd} / \mathrm{kg}]$ & $k_{\text {eff }}$ & \multicolumn{2}{c}{ Difference } & & $k_{\text {eff }}$ & \multicolumn{2}{c}{ Difference } \\
\hline & Spline & TRANSX & TRANSX & & Spline & TRANSX & TRANSX \\
& $15 \mathrm{pts}$ & $15 \mathrm{pts}$ & $7 \mathrm{pts}$ & & $15 \mathrm{pts}$ & $15 \mathrm{pts}$ & $7 \mathrm{pts}$ \\
\hline 0.00 & 1.3259 & -0.0058 & -0.0151 & & 1.2784 & -0.0002 & -0.0006 \\
5.84 & 1.1952 & -0.0050 & -0.0133 & & 1.1545 & -0.0003 & -0.0011 \\
10.51 & 1.1393 & -0.0044 & -0.0120 & & 1.1034 & -0.0003 & -0.0014 \\
15.18 & 1.0917 & -0.0038 & -0.0103 & & 1.0611 & -0.0003 & -0.0016 \\
19.85 & 1.0494 & -0.0030 & -0.0084 & & 1.0244 & -0.0004 & -0.0018 \\
24.52 & 1.0112 & -0.0021 & -0.0064 & & 0.9920 & -0.0004 & -0.0018 \\
28.03 & 0.9847 & -0.0015 & -0.0049 & & 0.9699 & -0.0004 & -0.0018 \\
\hline
\end{tabular}

\section{EFFECT ON REACTOR SIMULATION}

To see the effect of interpolation on reactor simulation, consider a simple problem run in the Jaguar/Bengal/Lynx code suite, that of a two-dimensional reflected pin-cell, using the geometry and materials specifications taken from the Consortium for Advanced Simulation of Light Water Reactors (CASL) core physics benchmark progression problems [9]. Unlike in the first progression problem, this pin-cell is depleted to show the effect of interpolation with burnup.

Three interpolation schemes are considered: TRANSX-style interpolation with a 7-point dilution grid, TRANSX-style interpolation with a 15-point dilution grid, and cubic spline with inverselinear interpolation with a 15-point dilution grid. The eigenvalue differences between these simulations is shown in Table 2. At beginning of life, the two interpolation schemes on the 15-point grid differ by approximately $580 \mathrm{pcm}$. The 7-point dilution grid differs from the 15-point dilution grid with splines by $1.5 \%$ in eigenvalue. These are excessively large errors, exaggerated by U-238's background cross section being approximately $50 \mathrm{~b}$, which is near the maximum error displayed in Fig. 2.

Next, the Dancoff factor is artificially adjusted to force U-238's background cross section to be approximately $100 \mathrm{~b}$, which is a base point in both dilution grids used. The eigenvalue differences are shown in Table 2. Beginning of life eigenvalue differences are only approximately $20 \mathrm{pcm}$ and $60 \mathrm{pcm}$. This suggests that a carefully selected dilution grid could, as much as the interpolation scheme, improve the eigenvalue and U-238's reaction rate. However, transuranics generated through depletion will have background cross sections that vary continuously with life, thus requiring accurate interpolation. A study of transuranic reaction rates as a function of interpolation scheme is essential to select a scheme for production use, but one is not shown here for the sake of brevity. 


\section{CONCLUSIONS}

This study investigated methods to accurately interpolate multigroup cross sections as a function of the background cross section, which was performed during the development of the Bengal code as a replacement for TRANSX. It was found that unacceptably high interpolation errors, which can lead to significant eigenvalue error in reactor simulation, can be found using a naive interpolation approach. By using a suitable interpolant, a 15-point dilution grid was found to lead to an acceptable error level without the drawbacks of larger grids. An example 15-point grid was shown here, but it was shown to be a poor choice for commercial light water reactor analysis. Regarding the interpolant function, rather than the log-linear interpolation used by TRANSX, cubic Hermite splines were shown to do a much better job of capturing the dilution dependence of cross sections. For the highest dilution values, the inverse-linear interpolation of TRANSX was retained.

\section{ACKNOWLEDGMENTS}

This work has been authored by Fluor Marine Propulsion, LLC, under Contract No. DOE-89233018CNR000004 with the U.S. Department of Energy. The United States Government retains and the publisher, by accepting this article for publication, acknowledges that the United States Government retains a non-exclusive, paid-up, irrevocable, and world-wide license to publish, distribute, translate, duplicate, exhibit, and perform the published form of this manuscript, or allow others to do so, for United States Government purposes.

\section{REFERENCES}

[1] S. J. Douglass and N. A. Gibson. "Bengal: A Multigroup Cross Section Processing Code for Large-Scale Calculations." In Transactions of the American Nuclear Society, Winter Meeting. Washington, D.C. (2019).

[2] A. M. Watson, R. E. Grove, and M. T. Shearer. "Effective Software Design for a Deterministic Transport System." Transactions of the American Nuclear Society, (97), pp. 482-484 (2007).

[3] R. E. MacFarlane. “TRANSX 2: A Code for Interfacing MATXS Cross-Section Libraries to Nuclear Transport Codes." Technical report, Los Alamos National Laboratory (1993).

[4] N. A. Gibson. Novel Resonance Self-Shielding Methods for Nuclear Reactor Analysis. Ph.D. thesis, Massachusetts Institute of Technology (2016).

[5] A. Hébert. Applied Reactor Physics. Presses Internationales Polytechnique (2010).

[6] R. E. MacFarlane and A. C. Kahler. "The NJOY Nuclear Data Processing System, Version 2012.” Technical Report LA-UR-12-27079, Los Alamos National Laboratory (2012).

[7] D. Knott and A. Yamamoto. Lattice Physics Computations. Springer, Boston, MA (2010). In: D. G. CACUCI (eds.), Handbook of Nuclear Engineering.

[8] R. Stamm'ler and C. Abbate. Methods of Steady-State Reactor Physics in Nuclear Design. Academic Press (1983).

[9] A. T. Godfrey. "VERA Core Physics Benchmark Progression Problem Specifications." Technical Report CASL-U-2012-0131-004 (2014). 